\title{
Can IDO activity predict primary resistance to anti-PD-1 treatment in NSCLC?
}

\author{
Andrea Botticelli ${ }^{1 *}$, Bruna Cerbelli ${ }^{2}$, Luana Lionetto ${ }^{3}$, Ilaria Zizzari ${ }^{4}$, Massimiliano Salati ${ }^{5}$, Annalinda Pisano ${ }^{2}$, \\ Mazzuca Federica ${ }^{1}$, Maurizio Simmaco ${ }^{6}$, Marianna Nuti ${ }^{4}$ and Paolo Marchetti ${ }^{1}$
}

\begin{abstract}
Background: Immune checkpoint inhibitors have revolutionized the treatment paradigm of highly lethal malignancies like advanced non-small cell lung cancer (NSCLC), demonstrating long-term tumour control and extended patient survival. Unfortunately, only $25-30 \%$ of patients experience a durable benefit, while the vast majority demonstrate primary or acquired resistance. Recently, indoleamine 2,3-dioxygenase (IDO) activity has been proposed as a possible mechanism of resistance to anti-PD-1 treatment leading to an immunosuppressive microenvironment.

Methods: Pre-treatment serum concentrations of tryptophan (trp) and kynurenine (kyn) were measured by high-performance liquid chromatography tandem mass spectrometry in NSCLC patients treated with second-line nivolumab. The IDO activity was expressed with kyn/trp ratio. The associations between kyn/trp ratio and early progression, performance status (PS), age, sex, brain metastases, pleural effusion, progression free survival (PFS) and overall survival (OS) were analyzed using Spearman test and Mann-Whitney test.
\end{abstract}

Results: Twenty-six NSCLC patients were included in our study; 14 of them (54\%) presented early progression ( $<3$ months) to nivolumab treatment. The median value of $\mathrm{kyn} / \mathrm{trp}$ ratio was $0.06 \mu \mathrm{g} / \mathrm{ml}$ and the median value of quinolinic acid was $68.45 \mathrm{ng} / \mathrm{ml}$. A significant correlation between early progression and higher kyn/trp ratio and quinolinic acid concentration was observed ( $p=0.017$ and $p=0.005$, respectively). Patients presenting lower values of kyn/ trp ratio and quinolinic acid levels showed longer PFS (median PFS not reached versus 3 months; HR: $0.3 ; p=0.018$ ) and OS (median OS not reached vs 3 months; HR: 0.18; $p=0.0005$ ).

Conclusion: IDO activity, expressed as kyn/trp ratio, is associated with response to immunotherapy; in particular, higher kyn/trp ratio could predict resistance to anti-PD-1 treatment. These preliminary results suggest the possibility of using anti-PD-1 plus IDO inhibitor in those patients with high level of kyn/trp ratio.

Keywords: IDO, Immunotherapy, Nivolumab, Kynurenine, Anti-PD-1

\section{Background}

In NSCLC, nivolumab showed a long-term benefit in a significant proportion of pretreated patients with 2-year overall survival (OS) of 23 and $29 \%$ in squamous and nonsquamous histology, respectively, over-performing standard chemotherapy $[1,2]$. However, more than two-thirds of NSCLC patients demonstrate primary or acquired resistance to $\mathrm{PD}-1$ inhibition resulting in treatment

\footnotetext{
*Correspondence: andreabotticelli@hotmail.it

1 Department of Clinical and Molecular Medicine, Sant'Andrea Hospital, Sapienza University of Rome, Via di Grottarossa 1035-1037, 00189 Rome, Italy

Full list of author information is available at the end of the article
}

failure [3]. Moreover, recent evidence suggested the existence of a novel pattern of disease progression referred to as hyper-progressive and characterized by a two-fold increase in tumour burden at first instrumental assessment following anti-PD-1 treatment and very poor OS [4]. Therefore, there is an urgent need for biomarkers able to predict treatment responsiveness and to identify those patients unlikely to respond to PD-1 blockade in order to candidate them to different treatment approaches. While some predictive factors of response to immune checkpoint inhibitors (ICI) have been proposed, among which PD-L1 expression/amplification, high tumor mutational burden, and mismatch repair gene defects, reliable 
biomarkers for treatment resistance are still lacking [3]. Indoleamine 2,3-dioxygenase (IDO) is a key enzyme catalyzing the first and rate-limiting step along the kynurenine (kyn) pathway of tryptophan (trp) metabolism outside the liver, which converts the essential amino acid l-trp to the main metabolite kyn [5]. Indoleamine 2,3-dioxygenase has been shown to act as immune checkpoint involved in peripheral immune tolerance since it is able to inhibit T-cells proliferation by starving them from trp to sensitize T-cells to apoptosis [6-11]. In NSCLC, augmented trp catabolism, resulting in higher kyn serum concentration, has been linked to more advanced stage at diagnosis, poorer prognosis and to a lesser likelihood of response to chemotherapy [12-14]. More interestingly, growing preclinical evidence suggests that increase in IDO activity can be involved in resistance to checkpoint inhibition [15] In the present study, we aimed to investigate whether IDO activity, expressed as kyn/trp ratio, can inform about response to nivolumab, particularly identifying those patients resistant to PD-1 blockade in a cohort of previously treated NSCLC.

\section{Methods \\ Patients}

Patients with stage IV NSCLC followed at our Institution from June 2016 to July 2017 were enrolled onto this retrospectively study. Inclusion criteria were: age $>18$ years; histologically-documented diagnosis of NSCLN; Eastern Cooperative Oncology Group (ECOG) performance status $\leq 2$; second-line treatment with anti-PD-1 nivolumab; adequate cardiac, pulmonary, renal, liver and bone marrow function; written informed consent. Exclusion criteria were: autoimmune disease; symptomatic interstitial lung disease and any other significant comorbidity; systemic immunosuppression; prior treatment with immune-stimulatory antitumor agents including checkpoint-targeted agents.

Nivolumab treatment was administered at a standard dose of $3 \mathrm{mg} / \mathrm{kg}$ every 2 weeks until disease progression or development of unacceptable toxicity. Radiological response was assessed with i-RECIST Criteria and classified according to disease control (complete response, partial response and stable disease) and progressive disease. All toxicity was graded according to the National Cancer Institute Common Terminology Criteria for Adverse Events (version 4.0) and toxicity assessments performed at day 1 of every cycle until the end of treatment. Progression-free survival (PFS) was defined as the time from patient registration on clinical trial until the first documented tumour progression or death from any cause. Overall survival (OS) was defined as the time from patient registration to death from any cause.
We defined as early progressors, patients experiencing disease progression within three months from the beginning of nivolumab. The study was conducted in accordance with good clinical practice guidelines and the declaration of Helsinki. The final version of the protocol was approved by the Institutional Ethics Committee.

\section{Kynurenine analysis}

We evaluated serum levels of trp, kyn, and quinolinic acid by a modified liquid chromatography-tandem mass spectrometry (LC-MS/MS) method, [ADHD]. Serum samples were collected and stored at $-80^{\circ} \mathrm{C}$ until analysis. Fifty $\mu \mathrm{l}$ of serum samples were deproteinized using $50 \mu \mathrm{l}$ of Internal Standard (IS) solution $(50 \mu \mathrm{M}$ in TCA $4 \%)$, vortex-mixed and centrifuged at $14,000 \mathrm{rpm}$ for $15 \mathrm{~min}$. Twenty $\mu \mathrm{l}$ of clean upper layer were injected into chromatographic system. Chromatographic separation of analytes was performed using an Agilent Liquid Chromatography System series 1100 (Agilent Technologies, USA), on a biphenyl column $(100 \times 2.1 \mathrm{~mm}$, Kinetex $2.6 \mu \mathrm{m}$ Biphenyl, $100 \AA$, Phenomenex, CA, USA) equipped with a security guard precolumn (Phenomenex, Torrance, CA, USA). The mobile phase consisted of a solution of $0.1 \%$ aqueous formic acid (A) and $100 \%$ methanol (B); elution was performed at flow rate of $400 \mu \mathrm{l} /$ min, using an elution gradient. The mass spectrometry method was performed on a 3200 triple quadrupole system Applied Biosystems, Foster City, CA, USA) equipped with a Turbo Ion Spray source. The detector was set in the positive ion mode. The instrument was set in the Multiple Reaction Monitoring (MRM) mode. Data were acquired and processed by the Analyst 1.5.1 Software.

\section{Statistical analysis}

In the descriptive analysis, quantitative variables were described as mean and range, while qualitative variables as number and percentage.

PFS and OS were estimated using the Kaplan-Meier method, comparison between groups were made using the log-rank test, and Mantel-Cox method was used to generate hazard ratios (HRs) and 95\% confidence intervals (CIs).

Correlation was estimated using Spearman's rho and comparison was evaluated using the nonparametric Mann-Whitney U test.

To determinate factors associated with early progressors, multivariate logistic regression models were used. Results of multivariate analysis were expressed in odds ratio and 95\% CIs. Statistical significance was set at $\mathrm{p}<0.05$. Statistical analyses were performed using IBM SPSS Statistics for Window Version 23.0 (Armonk, NY, USA) or GraphPad Prism (GraphPad, Inc, San Diego, CA, USA). 


\section{Results}

\section{Clinical characteristics}

Twenty-six stage IV NSCLC patients treated with second-line nivolumab were enrolled in this study. Baseline clinicopathological characteristics of patients are summarized in Table 1. Among them, 19 patients had

Table 1 Main baseline characteristics of the study population

\begin{tabular}{|c|c|c|c|}
\hline & $\mathrm{N}(\%)$ & $\begin{array}{l}\text { Serum kyn/ } \\
\text { trp ratio }(\mu \mathrm{g} / \\
\mathrm{ml})\end{array}$ & $\begin{array}{l}\text { Serum quinolic acid } \\
\text { concentration ( } \mathrm{ng} / \\
\mathrm{ml} \text { ) }\end{array}$ \\
\hline \multicolumn{4}{|l|}{ Age } \\
\hline$<65$ & $11(42.3)$ & $0.06 \pm 0.03$ & $74.47 \pm 45.93$ \\
\hline $65+$ & $15(57.7)$ & $0.08 \pm 0.05$ & $102.55 \pm 72.99$ \\
\hline \multicolumn{4}{|l|}{ Sex } \\
\hline Male & $20(76.9)$ & $0.08 \pm 0.04$ & $99.21 \pm 69.37$ \\
\hline Female & $6(23.1)$ & $0.05 \pm 0.02$ & $62.22 \pm 24.24$ \\
\hline \multicolumn{4}{|l|}{ Histology } \\
\hline Adenocarcinoma & $6(23.1)$ & $0.09 \pm 0.04$ & $127.85 \pm 59.78$ \\
\hline $\begin{array}{l}\text { Squamous-cell } \\
\text { carcinoma }\end{array}$ & $19(73.1)$ & $0.07 \pm 0.04$ & $79.69 \pm 63.25$ \\
\hline $\begin{array}{l}\text { Undifferentiated } \\
\text { tumor }\end{array}$ & $1(3.8)$ & 0.04 & 76.2 \\
\hline \multicolumn{4}{|l|}{ ECOG PS } \\
\hline $0-1$ & $25(96.2)$ & $0.07 \pm 0.03$ & $88.26 \pm 63.54$ \\
\hline$>1$ & $1(3.8)$ & 0.18 & 151 \\
\hline \multicolumn{4}{|l|}{ Cerebral metastasis } \\
\hline Yes & $3(11.5)$ & $0.05 \pm 0.04$ & $91.54 \pm 64.77$ \\
\hline No & $23(88.5)$ & $0.08 \pm 0.04$ & $84.03 \pm 64.14$ \\
\hline \multicolumn{4}{|l|}{ Pleural effusion } \\
\hline Yes & $6(23.1)$ & $0.07 \pm 0.04$ & $79.24 \pm 45.54$ \\
\hline No & $20(76.9)$ & $0.09 \pm 0.05$ & $110.39 \pm 99.95$ \\
\hline \multicolumn{4}{|l|}{ Early progressor } \\
\hline Yes & $14(53.8)$ & $0.09 \pm 0.05$ & $121.15 \pm 72.34$ \\
\hline No & $12(46.2)$ & $0.05 \pm 0.01$ & $55.12 \pm 20.18$ \\
\hline
\end{tabular}

squamous-cell carcinoma and the remaining had nonsquamous histology (6 adenocarcinoma and 1 undifferentiated tumour). Twenty patients were male (76.9\%), and median age was 65 years. The median value of kyn/ trp was $0.06 \mu \mathrm{g} / \mathrm{ml}$ and median value of quinolinic acid was $68.45 \mathrm{ng} / \mathrm{ml}$.

\section{Correlation of serum kyn/trp ratio and quinolinic acid concentration with immunotherapy response}

With a median follow up of 6.9 months, 7 (27\%), 5 (19\%) and $14(54 \%)$ patients had a stable disease (SD), a partial response (PR) and a progressive disease (PD), respectively, according to i-RECIST v1.1 criteria. All patients were assessed at baseline for serum trp, kyn and quinolinic acid levels. Patients who showed an early progression (within 3 months) to nivolumab $(n=14)$ had a significantly higher concentration of kyn/trp ratio than others (0.09 vs 0.05 , respectively, $p=0.01)$ as well as a higher quinolinic acid level $(121,15$ vs 55,12 , respectively, $p=0.01$ ) (Fig. 1).

On multivariate analysis (Table 2), kyn/trp ratio was significant associated with early progression $(p=0.01)$. We observed a significant correlation only between early progression and kyn/trp ratio and quinolinic acid concentration (respectively $p=0.017$ and $p=0.005$ ).

\section{Correlation of serum kyn/trp ratio and quinolinic acid concentration with survival}

Median PFS was 4 months and median OS was 6.5 months in the whole cohort.

Patients were stratified by median value of serum $\mathrm{kyn} / \mathrm{trp}$ value $(0.06 \mu \mathrm{g} / \mathrm{ml})$ and of serum quinolinic acid concentration $(68.45 \mathrm{ng} / \mathrm{ml})$. PFS was significantly longer in patients presenting lower values of kyn/trp than in patients showing higher values of kyn/ trp (median PFS not reached versus 3 months; HR: 0.2 ; 95\% CI 0.06-0.62; $p=0.001)$. Similar results were
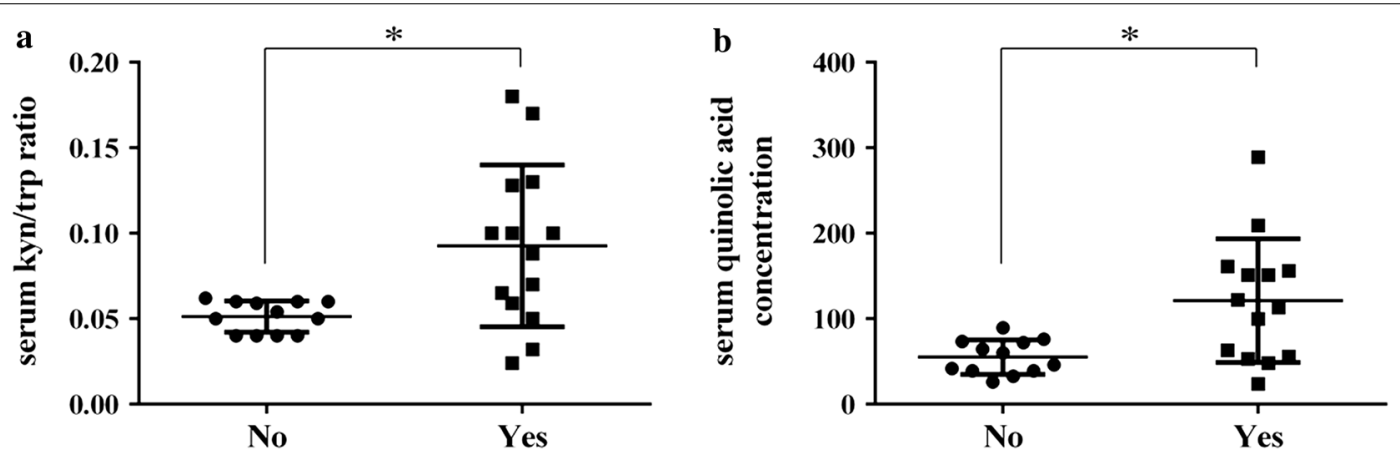

Fig. 1 Correlation of serum kyn/trp ratio and serum quinolic acid concentration with immunotherapy response. Patients who showed an early progression (YES, $n=14$ ) present a significantly higher concentration of kyn/trp ratio (a) and quinolic acid concentration (b) compared to patients who do not have an early progression $(\mathrm{NO}, \mathrm{n}=12)$. \#p $<0.05$ (Mann-Whitney test) 
Table 2 Multivariate analysis: association between patiens characteristics and early progression

\begin{tabular}{lll}
\hline & p value & Odds ratio $(\mathbf{9 5} \% \mathbf{C l})$ \\
\hline Cerebral metastasis & 0.48 & $3.18(0.12-81.69)$ \\
Pleural effusion & 0.66 & $0.53(0.33-8.71)$ \\
ECOG PS & 0.39 & $2.58(0.92-22.75)$ \\
Serum kyn/trp & 0.01 & $0.01(0.00-0.41)$ \\
Serum quinolic acid & 0.81 & $1.38(0.09-20.30)$ \\
\hline
\end{tabular}

observed in patient stratified by quinolinic acid values (median PFS not reached versus median 3 months; HR: 0.3; CI 0.1-0.9; $p=0.018$ ) (Fig. 2a).

Patients with a lower kyn/trp ratio value showed even a significantly better OS compared with patients with a higher kyn/trp ratio value (median OS not reached versus median 3 months; HR 0.18; 95\% CI $0.06-0.56 ; p=0.0005)$. Similar results were observed in patient stratified by quinolinic acid values (median OS not reached versus median 3 months; HR 0.2; 95\% CI 0.06-0.660; $p=0.0077$ ) (Fig. 2b).

\section{Discussion}

In our preliminary analysis we found that baseline serum kyn/trp ratio, expression of IDO activity, could represent both a prognostic and predictive value in advanced NSCLC patients treated with second-line nivolumab. In fact, lower baseline level of kyn/trp ratio were significantly associated with better PFS and OS in our cohort. More interestingly and conversely, higher level of kyn/ trp ratio correlated with early progression retaining an independent statistically significant value in multivariate analysis. In this study, patients were selected in accordance with eligibility criteria from registrative clinical trials of nivolumab in pretreated NSCLC (CheckMate 017 and CheckMate 057) and median PFS was consistent with historical data reported in the literature $[16,17]$.

Given the evidence that only a minority of cancer patients can benefit from ICI, a large amount of research is currently focused on defining predictive biomarkers able to select responsive or resistant patients in order to maximize the cost-effectiveness of treatment.

Over last few years, several predictive factors have been proposed that rely on patients' characteristics as well as tumour and microenvironment profiles. PD-L1, which is expressed both on tumour and inflammatory cells, is arguably the most extensively studied biomarker for ICIs responsiveness, although it has showed some limitations [3]. More recently, the understanding of the

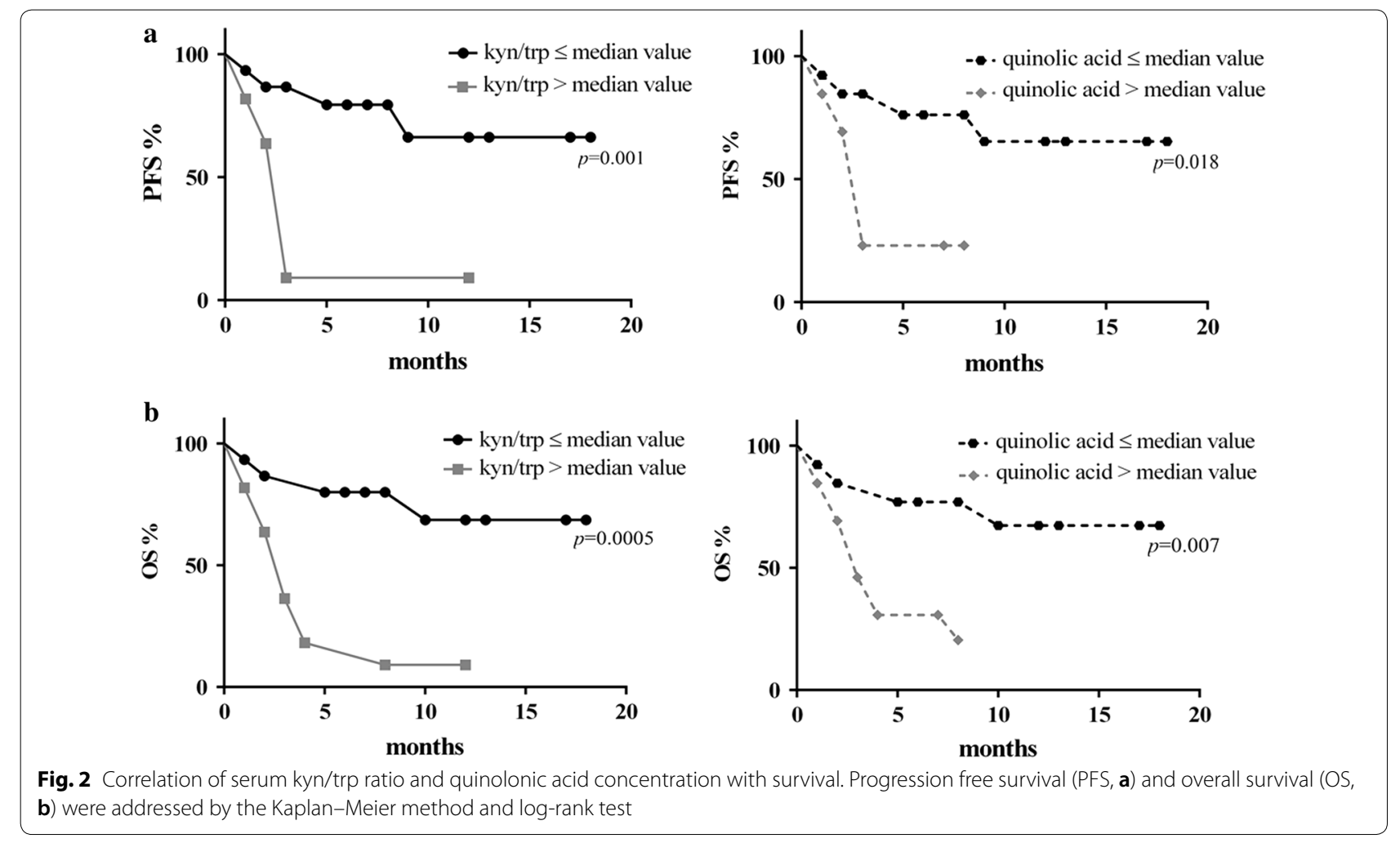


correlation of microsatellite instability status with immunotherapy response has led to the FDA cancer site/histology-agnostic approval of the anti-PD-1 pembrolizumab [18]. Accordingly, tumour mutational burden (TMB) and mRNA profile are being considered promising response indicators [19]. In particular TMB has been used to select patients in CHECKMATE 227 trial, demonstrating a benefit of immunotherapy in patients with mutational load higher than 10 mutations per megabase. Regarding circulating biomarkers, some encouraging results have been reported for IL 8 [20] and circulating T lymphocytes [21]. However, all these putative biomarkers have strong limitations and they have not shown clinical usefulness for the different settings.

In this context, IDO has been identified as a marker of activation of immunological pathways induced by IFNgamma, representing one of the main immune escape mechanism of tumor cells. IDO activity would also appear as negative prognostic factor in stage III-IV lung cancer [22-27].

Furthermore, Allison et al. demonstrated that IDO knockout mice treated with anti CTLA-4, anti PD-1 or anti GITR presented delay in B16 melanoma tumour growth and increased OS compared with wild-type mice [28]. Notably, tissue expression of IDO has been lately associated with a better response to nivolumab in patients with renal cell carcinoma [29].

Our preliminary results showed that kyn/trp ratio serum levels are statistically higher in early progressors allowing to identify a proportion of NSCLC patients with intrinsic resistance to anti PD-1, and with a poor prognosis both in terms of PFS and OS. In particular, the median PFS of the whole cohort is not reached, while the median PFS of "high kyn/trp ratio patients "was 3 months, very similar to that of hyperprogressive patients from the study by Champiat et al. [4]. Interestingly, in our cohort, the IDO activity does not seem to be correlated with clinical parameters such as brain metastases, pleural effusion, age and ECOG PS.

Moreover, quinolinic acid levels are strongly associated with response to nivolumab in our study. Quinolinic acid reflects the metabolism of kynurenine and it is involved in tumour progression not only diminishing immune activation but also increasing the synthesis of NAD, through quinolinic acid phosphoribosyltransferase, that is necessary to prevent apoptosis of neoplastic cells [30].

Our findings suggest a possible role to IDO in mediating resistance to anti $\mathrm{PD}-1$ agents and provide a rationale for an early administration of anti-IDO compounds in those patients with high baseline kyn/trp ratio or high quinolinic acid levels. In fact, patient selection based on this biomarker could most probably avoid negative results of clinical trials designed without considering the immunological background of patients, i.e. the extent of the immunosuppressive milieu.

\section{Conclusion}

In conclusion we found that the kyn/trp ratio and quinolinic acid level in the peripheral blood of NSCLC patients may be a potential predictive biomarker of resistance to anti-PD-1. The kyn/trp ratio and quinolinic acid are associated with early progression, PFS and OS. Despite the small sample, our study suggests that IDO could represent an easy-assessable and cost-effective biomarker particularly useful in identifying patients more likely to have a benefit with a combination of antiPD-1 and anti-IDO. Future clinical trials are warranted to confirm its role as predictive biomarkers as well as therapeutic target, introducing immuno-oncology in the scenario of precision immuno-oncology.

\section{Authors' contributions}

$A B, M N$ and $P M$ conceived and designed the work. $A B, B C, M S$, wrote the manuscript. FM, LL, IZ acquired the samples, performed the experiments and acquired the data. $A P$, analysed the data. $A B, P M, M S I$ discussed results and implications of findings. All authors read and approved the final manuscript.

\section{Author details \\ ${ }^{1}$ Department of Clinical and Molecular Medicine, Sant'Andrea Hospital, Sapienza University of Rome, Via di Grottarossa 1035-1037, 00189 Rome, Italy. ${ }^{2}$ Department of Radiological, Oncological and Pathological Sciences, Sapi- enza University of Rome, Rome, Italy. ${ }^{3}$ Experimental Immunology Laboratory, Biochemistry Laboratory, IDI-IRCCS FLMM, Rome, Italy. ${ }^{4}$ Department of Experi- mental Medicine, "Sapienza" University of Rome, Rome, Italy. ${ }^{5}$ Department of Oncology, Università di Modena e Reggio Emilia, Policlinico di Modena, Modena, Italy. ${ }^{6}$ Advanced Molecular Diagnostics Unit, Sant'Andrea Hospital, Sapienza University of Rome, Rome, Italy.}

\section{Acknowledgements}

Not applicable.

\section{Competing interests}

PAOLO MARCHETTI (PM) has/had a consultant/advisory role for BMS, RocheGenentech, MSD, Novartis, Amgen, Merck Serono, Pierre Fabre, Incyte. The other authors declare they have no competing interests.

\section{Availability of data and materials}

All data generated and analysed during the current study are included in this published article.

\section{Consent for publication}

All the patients provided written informed consent.

Ethics approval and consent to participate

This study was approved by a local ethics committee RIF. CE: 4421

Funding

This work was supported by Sapienza University of Rome.

\section{Publisher's Note}

Springer Nature remains neutral with regard to jurisdictional claims in published maps and institutional affiliations.

Received: 4 May 2018 Accepted: 2 August 2018

Published online: 06 August 2018 


\section{References}

1. Postow MA, Callahan MK, Wolchok JD. Immune checkpoint blockade in cancer therapy. J Clin Oncol. 2015:33(17):1974-82.

2. Horn L, Spigel DR, Vokes EE, et al. Nivolumab versus docetaxel in previously treated patients with advanced non-small-cell lung cancer: two-year outcomes from two randomized, open-label, phase III trials (CheckMate 017 and CheckMate 057). J Clin Oncol. 2017:35:3924-33.

3. Salati M, Baldessari C, Cerbelli B, Botticelli A. Nivolumab in pretreated non-small cell lung cancer: continuing the immunolution. Transl Lung Cancer Res. 2018. https://doi.org/10.21037/tlcr.2018.01.14.

4. Champiat S, Dercle L, Ammari S, Massard C, Hollebecque A, Postel-Vinay $\mathrm{S}$, et al. Hyperprogressive disease (HPD) is a new pattern of progression in cancer patients treated by anti-PD-1/PD-L1. Clin Cancer Res. 2017;23(8):1920-8. https://doi.org/10.1158/1078-0432.CCR-16-1741.

5. Badawy AA. Kynurenine pathway of tryptophan metabolism: regulatory and functional aspects. Int J Tryptophan Res. 2017;15(10):1178646917691938. https://doi.org/10.1177/1178646917 691938 (eCollection 2017. Review)

6. Mellor AL, Munn DH. Tryptophan catabolism and T-cell tolerance: immunosuppression by starvation? Immunol Today. 1999;20(10):469-73.

7. Munn DH, Shafzadeh E, Attwood JT, Bondarev I, Pashine A, Mellor AL. Inhibition of T cell proliferation by macrophage tryptophan catabolism. J Exp Med. 1999;189(9):1363-72.

8. Hwu P, Du MX, Lapointe R, Do M, Taylor MW, Young HA. Indoleamine 2,3-dioxygenase production by human dendritic cells results in the inhibition of T cell proliferation. J Immunol. 2000;164(7):3596-9.

9. Curti A, Pandolfi S, Valzasina B, et al. Modulation of tryptophan catabolism by human leukemic cells results in the conversion of CD25- into CD25+ T regulatory cells. Blood. 2007;109(7):2871-7.

10. Chen W, Liang X, Peterson AJ, Munn DH, Blazar BR. The indoleamine 2,3-dioxygenase pathway is essential for human plasmacytoid dendritic cell-induced adaptive T regulatory cell generation. J Immunol. 2008;181(8):5396-404.

11. Chung DJ, Rossi M, Romano E, et al. Indoleamine 2,3-dioxygenaseexpressing mature human monocyte-derived dendritic cells expand potent autologous regulatory T cells. Blood. 2009;114(3):555-63.

12. Uyttenhove C, Pilotte L, Thate I, Stroobant V, Colau D, Parmentier N, et al. Evidence for a tumoral immune resistance mechanism based on tryptophan degradation by indoleamine 2,3-dioxygenase. Nat Med. 2003;9:1269-74. https://doi.org/10.1038/nm934.

13. Zamanakou M, Germenis AE, Karanikas V. Tumor immune escape mediated by indoleamine 2,3-dioxygenase. Immunol Lett. 2007;111:69-75. https://doi.org/10.1016/j.imlet.2007.06.001.

14. Wang $Y$, Hu GF, Wang ZH. The status of immunosuppression in patients with stage III B or IV non-small-cell lung cancer correlates with the clinical characteristics and response to chemotherapy. OncoTargets Ther. 2017;10:3557-66.

15. Holmgaard RB, Zamarin D, Munn DH, Wolchok JD, Allison JP. Indoleamine 2,3-dioxygenase is a critical resistance mechanism in antitumor T-cell immunotherapy targeting CTLA4. J Exp Med. 2013;210(7):1389-402.
16. Brahmer J, Reckamp KL, Baas P, et al. Nivolumab versus docetaxel in advanced squamous-cell non-small-cell lung cancer. N Engl J Med. 2015:373:123-35

17. Borghaei $H$, Paz-Ares L, Horn L, et al. Nivolumab versus docetaxel in advanced non-squamous non-small-cell lung cancer. N Engl J Med. 2015;373:1627-39.

18. Lemery S, Keegan P, Pazdur R. First FDA approval agnostic of cancer site - When a biomarker defines the indication. N Engl J Med. 2017:377(15):1409-12. https://doi.org/10.1056/NEJMp1709968.

19. Hellmann MD, Ciuleanu TE, Pluzanski A. Nivolumab plus ipilimumab in lung cancer with a high tumor mutational burden. N Engl J Med. 2018;378(22):2093-104. https://doi.org/10.1056/NEJMoa1801946 (Epub 2018 Apr 16).

20. Sanmamed MF, Perez-Gracia JL, Fusco JP, et al. Changes in serum IL8 levels reflect and predict response to anti-PD-1 treatment in melanoma and non-small cell lung cancer patients. Ann Oncol. 2016;27(suppl_6):10550. https://doi.org/10.1093/annonc/mdw378.03.

21. Dronca RS, Liu X, Harrington SM, et al. T cell Bim levels reflect responses to anti-PD-1 cancer therapy. JCI Insight. 2016;1(6):e86014. https://doi. org/10.1172/jci.insight.86014.

22. Prendergast GC. Immune escape as a fundamental trait of cancer: focus on IDO. Oncogene. 2008;27(28):3889-900. https://doi.org/10.1038/ onc.2008.35 (Epub 2008 Mar 3).

23. Heng B, Lim CK, Lovejoy DB, et al. Understanding the role of the kynurenine pathway in human breast cancer immunobiology. Oncotarget. 2016;7:6506-20. https://doi.org/10.18632/oncotarget.6467.

24. Huang SC, Fanidi A, Ueland PM, et al. Circulating biomarkers of tryptophan and the kynurenine pathway and lung cancer risk. Cancer Epidemiol Prev Biomarkers. 2014;23:461-8.

25. Parra ER, Villalobos P, Zhang J, et al. Immunohistochemical and image analysis-based study demonstrate that several immune checkpoints are co-expressed in non-small cell lung carcinoma tumors. J Thorac Oncol. 2018. https://doi.org/10.1016/j.jtho.2018.03.002.

26. Austin CJ, Rendina LM. Targeting key dioxygenases in tryptophan-kynurenine metabolism for immunomodulation and cancer chemotherapy. Drug Discov Today. 2014;20:609-17.

27. Wang W, Huang L, Jin JY, et al. IDO immune status after chemoradiation may predict survival in lung cancer patients. Cancer Res. 2018;78:809-16.

28. Holmgaard RB, Zamarin D, Munn DH, Wolchok JD, Allison JP. Indoleamine 2,3-dioxygenase is a critical resistance mechanism in antitumor T cell immunotherapy targeting CTLA-4. J Exp Med. 2013;210(7):1389-402. https://doi.org/10.1084/jem.20130066 (Epub 2013 Jun 10).

29. Seeber A, Klinglmair G, Fritz J, et al. High IDO-1 expression in tumor endothelial cells is associated with response to immunotherapy in metastatic renal cell carcinoma. Cancer Sci. 2018. https://doi.org/10.1111/ cas. 13560 .

30. Sahm F, Oezen I, Opitz CA, Radlwimmer B, von Deimling A, et al. The endogenous tryptophan metabolite and NAD+ precursor quinolinic acid confers resistance of gliomas to oxidative stress. Cancer Res. 2013;73:3225-34

\footnotetext{
Ready to submit your research? Choose BMC and benefit from:

- fast, convenient online submission

- thorough peer review by experienced researchers in your field

- rapid publication on acceptance

- support for research data, including large and complex data types

- gold Open Access which fosters wider collaboration and increased citations

- maximum visibility for your research: over $100 \mathrm{M}$ website views per year
}

At BMC, research is always in progress.

Learn more biomedcentral.com/submissions 